\title{
Possible Correlation Effects of Surface State Electrons on a Solid Hydrogen Film
}

\author{
Frieder Mugele, Uwe Albrecht and Paul Leiderer \\ Fakultät für Physik, Universität Konstanz, FRG \\ Kimitoshi Kono \\ Institute for Solid State Physics, University of Tokyo, Roppongi, \\ Tokyo, 106 Japan
}

We have investigated the transport properties of surface state electrons on thin quench-condensed hydrogen films for various electron densities. The surface state electron mobility showed a continuous dependence on the plasma parameter $\Gamma$ in the range from 20 to 130 , indicating a strong influence of correlation effects within the electron system for all measured values of $\Gamma$.

Surface state electrons (SSE) on cryogenic surfaces such as helium, hydrogen and neon have been receiving continuous attention over the past years ${ }^{1}$. They have been used as surface probes ${ }^{2,3}$, but there is also major interest in the properties of the 2-dimensional electron system itself. High electron densities can be reached on solid substrates which furthermore allow to study the effects of varying electron conductivity within the system by changing the roughness of the substrate ${ }^{2,4}$. We have used thin, quench-condensed solid hydrogen substrates. Thermal annealing lowers their roughness, rendering the film sufficiently smooth to reach high electron mobilities ${ }^{5}$. In this paper we report on pronounced correlation effects within the SSE system on a solid hydrogen film over a wide range of electron densities.

We prepared $\sim 1 \mu \mathrm{m}$ thin hydrogen films by quench-condensation at $1.8 \mathrm{~K}$ onto a float glass plate and subsequent annealing (see ref.5 for a description of the annealing procedure). Before charging the film surface a small amount of helium gas was admitted to the cell in order to slow down the electrons and thus prevent them from penetrating the hydrogen film. The electron density was controlled by the applied electric holding field. 

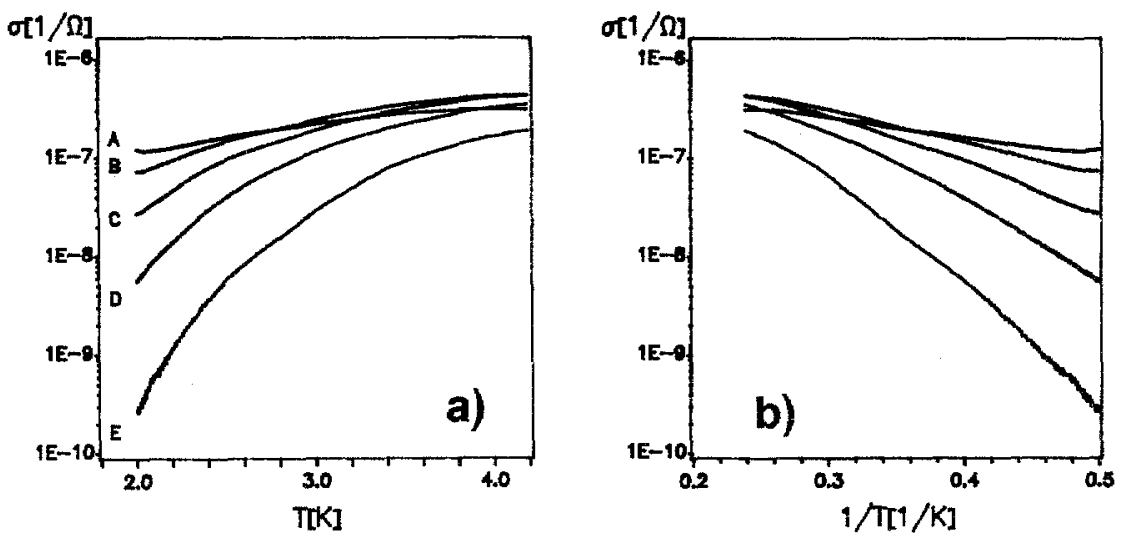

Figure 1: (a) SSE conductivity on an annealed, quench-condensed hydrogen film as a function of temperature for various electron densities $n_{s}, A: 0.051$ B:0.20 C:0.46 D:0.81 E:1.27 $\left[10^{10} \mathrm{~cm}^{-2}\right]$, (helium gas atom density $n_{g}=2.0 \times 10^{20} \mathrm{~cm}^{-3}$ ). (b) Arrhenius plot of the same data.

We measured the ac-conductivity of the electron system at a frequency of $50 \mathrm{kHz}$ using the Sommer-Tanner technique ${ }^{6}$. Details of the experimental setup are given elsewhere ${ }^{2}$.

Figure 1a shows the SSE conductivity vs. temperature for a range of electron densities $n_{s}$. The substrate was identical for all runs. There was helium gas with a gas atom density of $n_{g}=2.0 \times 10^{20} \mathrm{~cm}^{-3}$ remaining in the cell. All data exhibit a decrease of the conductivity towards the low temperature end of the measurements. This effect is more pronounced for higher electron densities, showing up in larger slopes of the corresponding Arrhenius plots (fig.1b). We also see that the absolute conductivities for high $n_{s}$ fall below the values obtained for low densities. This is surprising at the first glance, since for a non-interacting electron system one would expect a linear rise of the conductivity $\sigma=e n_{s} \mu$ ( $e$ : electron charge) with $n_{s}$, as long as the electron mobility $\mu$ is constant. However, increasing the electron density can lead to additional effects: correlation within the electron system can influence the mobility and the increased electric holding field can lead to a localization of electrons at surface inhomogeneities.

Assuming the number of mobile electrons to be constant we can estimate the mobility $\mu$ from our data by $\mu=\sigma / n_{s} e$ (on our hydrogen films $\mu$ was still too low for an accurate determination with a magnetoconductivity measurement). In figure 2 we plot the mobility vs. the plasma parameter $\Gamma=\sqrt{\pi n_{s}} e^{2} / \epsilon k_{B} T$ ( $\epsilon$ : dielectric constant of the surrounding medium, $k_{B}$ : Boltzmann constant) which characterizes the relation between electronic correlations and thermal excitations within the system. Surprisingly all the data collaps on a single continuous line (the bending down at the 


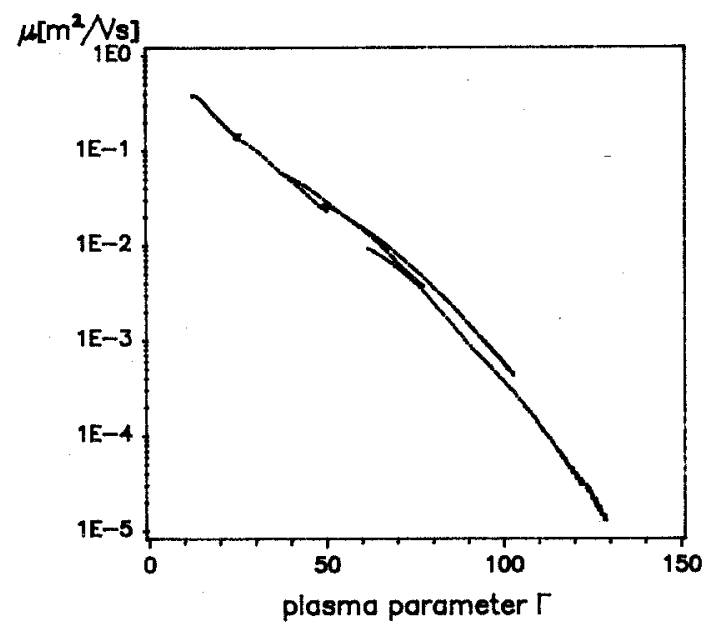

Figure 2: Data of fig.1 plotted as mobility $\mu$ vs. plasma parameter $\Gamma$.

high temperature, i.e. low $\Gamma$ end of each run is due to a layering of the helium gas adsorbing on the hydrogen surface $\left.{ }^{5}\right)$. The overall temperature dependence of the SSE mobility thus appears to be due to the increasing importance of correlation among the electrons at lower temperature. This agrees qualitatively with a detailed study of correlation effects in SSE on solid neon by Kajita?

Attempts to explain the data in terms of single electron effects are not satisfactory. In an earlier work the observed Arrhenius-type behavior (fig.1b) was interpreted as being due to thermal activation of the electrons from potential wells resulting from a surface roughness of the hydrogen substrate. This would lead to an effective electron density $n_{\text {eff }}=n_{s} \exp (-V / k t)$ contributing to the conductivity, where $V$ is some average depth of the potential wells. Accordingly, $V$ should be a function of the applied holding field. However, experiments with varying holding field at fixed electron density show the field influence to be quite weak, thus ruling it out as an explanation. Localization of the electrons in the helium gas atmosphere above the substrate ${ }^{8}$ also leads to a thermal activation-type behavior $\sigma_{\text {eff }}=\sigma_{o} \exp \left(-\gamma n_{g} / T\right)$ (the constant $\gamma$ results from single electron scattering from fluctuations of $n_{g}$ ). The strong $n_{s}$-dependence of the supposed activation energies in our data (fig.1b) reflects the breakdown of this single electron picture.

The decrease of $\sigma$ with the plasma parameter in fig. 2 gets more pronounced towards high $\Gamma$; the electron system is expected to undergo a transition to a Wigner-crysta ${ }^{9}$ around $\Gamma=140$, a value we do not quite reach in these experiments. It is known from other experiments ${ }^{2}$ that the slope of data like in fig. 2 depends on the degree of surface roughness of 
the hydrogen substrate. Thus we conclude that the correlation effect we observe is in fact mediated by surface inhomogeneities. Already few electrons pinned at such surface defects suffice to hamper the mobility of the surrounding electrons. Their influence is expected to increase with the correlation within the system, at the onset of Wigner-crystallisation the electrons should be completely locked. Efforts toward a more quantitative understanding of the underlying mechanism are underway.

In conclusion, we have measured the temperature dependent conductivity of surface state electrons on a quench-condensed solid hydrogen substrate for a wide range of electron densities $n_{s}$. We find that the electron mobility $\mu$ is a continuous function of only the plasma parameter $\Gamma$, in our experiments ranging from 10 to 130 . Thus the dependence of $\mu$ on $n_{s}$ and temperature appears to be almost exclusively due to correlations within the electron system. Surface roughness of the hydrogen substrate is believed to couple the correlation to the electron mobility.

This work was supported by the SFB306 of the Deutsche Forschungsgemeinschaft. One of us (K.K.) appreciates financial support by the Alexander von Humboldt-Stiftung.

\section{References}

1. P.Leiderer, Proceedings of the International School on Quantum Solids, Liquids and Gases, Elba, June 19-30, 1991, to be published in J.Low Temp.Phys., and references therein

2. K.Kono, U.Albrecht and P.Leiderer, J.Low Temp.Phys. 82, 279 (1991)

3. D.Cieslikowski, A.J.Dahm and P.Leiderer, Phys.Rev.Lett. 58, 1751 (1987)

4. P.A.Adams and M.A.Paalanen, Phys.Rev.Lett. 58, 2106 (1987)

5. U.Albrecht, P.Leiderer and K.Kono, in Proceedings of the International Symposium on Dynamical Processes on Solid Surfaces, Tokyo, April 20-23, 1992, to be published in Surf.Sci.

6. W.T.Sommer and D.J.Tanner, Phys.Rev.Lett. 27, 1345 (1971)

7. K.Kajita, Y.Nishio and W.Sasaki, Surf.Sci. 170, 88 (1986)

8. P.A.Adams and M.A.Paalanen, Phys.Rev.Lett. 61, 451 (1988); P.A.Adams, Phys.Rev.Lett. 65, 3333 (1990)

9. C.C.Grimes and G.Adams, Phys.Rev.Lett. 42, 795 (1979) 